\title{
THE FORMAL STRUCTURE OF A DENUMERABLE SYSTEM
}

\author{
BY \\ R. L. GOODSTEIN
}

Introduction. A strictly formal mathematical system comprising definite and variable number and function signs, logical constants and universal and existential operators, and employing the sentential calculus, may be termed classical if the application of the operators to the variables is without restriction; as is well known classical analysis is formalisable in such a system [1] $\left(^{1}\right)$. If the application of the operators is restricted to variables whose range of values is a finite class, the system is said to be finitist. A limited part only of classical analysis can be formulated in a system finitist in this sense [2]. A denumerable system is one in which the universal and existential operators are restricted to variables whose range of values is a class of cardinal $\boldsymbol{\aleph}_{0}$. The concept of a denumerable system was introduced by Weyl [4]. The purpose of this paper is to indicate how wide a part of classical function theory may be formulated in a denumerable system.

Formal characterisation. The primary elements of the system are numerals, numeral variables and numeral functions, the universal, existential, and minimal operators, and the logical constants. The calculus of the primary elements will be taken to be the system $Z_{\mu}$ of Hilbert-Bernays [1], so that $a t$ the first level, a denumerable system differs in no respect from the classical. (In particular the tertium non datur is valid in the form . $(A x)(f(x)=0) . \vee \cdot(E x)$ $\sim(f(x)=0)$., where $x$ is a numeral variable.) We use different kinds of variables to distinguish numeral variables from function variables (a denumerable infinity of both kinds being included amongst the primary elements) and we rely on Russell's ramified theory of types [5] for avoiding paradoxes.

The secondary elements are integral and rational number and function signs, which may be eliminated from the formulas of the system by means of the following definitions:

An integer is a pair of numerals $[p, q]$; we define $[p, q] \geqq\left[p^{\prime}, q^{\prime}\right],[p, q]$ $<\left[p^{\prime}, q^{\prime}\right]$ according as $p+q^{\prime} \geqq p^{\prime}+q, p+q^{\prime}<p^{\prime}+q .[p, q]$ is called a positive or negative integer according as $p>q$ or $p<q$; it follows that $[p, q]$ is positive or negative according as $[p, q] \gtrless[0,0]$.

An integer function $f([p, q])$ is a pair of numeral functions $[P(p, q)$, $Q(p, q)]$ such that

Presented to the Society, December 29, 1949; received by the editors February 28, 1949 and, in revised form, August 15, 1949.

(1) Numbers in brackets refer to the bibliography at the end of the paper. 


$$
(A p)(A q)\left\{\cdot\left[p^{\prime}, q^{\prime}\right]=[p, q] . \rightarrow \cdot\left[P\left(p^{\prime}, q^{\prime}\right), Q\left(p^{\prime}, q^{\prime}\right)\right]=[P(p, q), Q(p, q)] .\right\},
$$

with an analogous formulation for integer functions of more argument places. For example, we define the sum, difference, product, and modulus functions by the conditions

$$
\begin{aligned}
\left(A p, q, p^{\prime}, q^{\prime}\right)\left\{\operatorname{Sum}\left([p, q],\left[p^{\prime}, q^{\prime}\right]\right)\right. & \left.=\left[p+p^{\prime}, q+q^{\prime}\right]\right\}, \\
\left(A p, q, p^{\prime}, q^{\prime}\right)\left\{\operatorname{Diff}\left([p, q],\left[p^{\prime}, q^{\prime}\right]\right)\right. & \left.=\left[p+q^{\prime}, p^{\prime}+q\right]\right\}, \\
\left(A p, q, p^{\prime}, q^{\prime}\right)\left\{\operatorname{Prod}\left([p, q],\left[p^{\prime}, q^{\prime}\right]\right)\right. & \left.=\left[p p^{\prime}+q q^{\prime}, p q^{\prime}+p^{\prime} q\right]\right\}, \\
(A p, q)\{(. p \geqq q . \rightarrow . \operatorname{Mod}[p, q] & =p-q .) \\
& \&(. p<q . \rightarrow . \operatorname{Mod}[p, q]=q-p .)\} .
\end{aligned}
$$

We define a rational number to be a pair of integers $\left[p_{1}, q_{1}\right] /\left[p_{2}, q_{2}\right], p_{2} \neq q_{2}$, such that $\left[p_{1}, q_{1}\right] /\left[p_{2}, q_{2}\right]=\left[p_{1}^{\prime}, q_{1}^{\prime}\right] /\left[p_{2}^{\prime}, q_{2}^{\prime}\right]$ if, and only if, Prod $\left(\left[p_{1}, q_{1}\right]\right.$, $\left.\left[p_{2}^{\prime}, q_{2}^{\prime}\right]\right)=\operatorname{Prod}\left(\left[p_{2}, q_{2}\right],\left[p_{1}^{\prime}, q_{1}^{\prime}\right]\right)$. It follows that $\left[p_{1}, q_{1}\right] /\left[p_{2}, q_{2}\right]$ $=\left[q_{1}, p_{1}\right] /\left[q_{2}, p_{2}\right]$, so that any rational number has an equal with positive second term. Denoting integers by $i, j$ with or without suffixes, and the sum and product functions by the usual abbreviations $i+j$, $i j$ we define, for positive integers $j, j^{\prime}, i / j \gtrless i^{\prime} / j^{\prime}$ according as $i j^{\prime} \gtrless i^{\prime} j$.

A rational function $f(i / j)$ is a pair of integer functions $I(i, j) / J(i, j)$ such that

$$
\left(i / j=i^{\prime} / j^{\prime}\right) \rightarrow\left\{I(i, j) / J(i, j)=I\left(i^{\prime}, j^{\prime}\right) / J\left(i^{\prime}, j^{\prime}\right)\right\}
$$

with an analogous formulation for functions of more than one argument place.

We take for granted a variety of common abbreviations such as 0 for $[0,0],+n$ for $[n, 0],-n$ for $[0, n],-[m, n]$ for $[n, m]$, single letters for rational numbers, and specialisations of the foregoing definitions, and so on.

Amongst the rational functions of a numeral variable we distinguish the "endless fractions with scale radix $\rho$ " (where $\rho$ is an arbitrarily chosen natural number greater than unity), which we shall call, for brevity, endless decimals; a positive endless decimal is a rational function $f(n) / \rho^{n}$ where $f(n)$ is a positive integer function such that, for all $n, 0 \leqq(f(n+1)-\rho f(n))<\rho$. If $f(n) / \rho^{n}$ is a positive endless decimal then $-f(n) / \rho^{n}$ is called a negative endless decimal.

For a non-negative rational $\xi=i / j$ we define $(\xi)_{n}=i(n) / \rho^{n}$, where $i(n)$ is the greatest integer not exceeding $\xi \rho^{n}$. If $\xi$ is negative then $(\xi)_{n}=-(-\xi)_{n}$.

Metamathematical signs. To describe the general theorems of the calculus we use the method of proof schemata, that is, we formulate general theorems and proofs in metamathematical function variables $f(x), f(n, x)$, and so on, which yield demonstrable formulae of the calculus when the function variables are replaced by definite function signs of the system. In particular we introduce function variables $(a)_{n},(b)_{n},(x)_{n},(y)_{n},(X)_{n}$ (with argument $n$ ) to express unspecified endless decimals.

To simplify the descriptions we employ also a variety of abbreviations 
including $a, b, x, y, X$ with, or without, suffixes and affixes, for endless decimals, and others listed below $(\$ \S 1.3,1.5,1.51)$.

1. Notation. If $x$ is a rational number or endless decimal such that, for some $k,(x)_{k}=0$, we write $x=0(k)$.

1.1. If $s(n)$ is a rational function, $x$ is said to be a limit of $s(n)$ if $(A k)(A n)(E p)\left\{s(n+p)-(x)_{k}=0(k)\right\}$.

1.2. A rational function $s(n)$ is convergent if $(A k)(E n)(A p)(s(n+p)-s(n)$ $=0(k))$.

1.3. The upper limit of a bounded positive rational function. Let $s(n)$ be a bounded positive rational function, so that $(A k)(E x)(A n)\left(x>\rho^{k} s(n)\right)$ holds. We define $a(k)$ explicitly by the equation

$$
a(k)=\mu_{x}(E m)(A n)\left((n \geqq m) \rightarrow x+1>\rho^{k} s(n)\right) ;
$$

then $a(k) \leqq \rho^{k} s(n)$ for infinitely many $n$, and $a(k)+1>\rho^{k} s(n)$ from some $n$ onwards. It follows that

$$
0 \leqq a(k+1)-\rho . a(k)<\rho
$$

and therefore $a(k) / \rho^{k}$ is a positive endless decimal; this decimal is called the upper limit of the function $s(n)$ and we write $a(k) / \rho^{k}=\lim \sup s(n)$. We observe that the upper limit lim sup $s(n)$ satisfies the condition 1.1 for a limit of the function $s(n)$, for

$$
(A k)(A m)(E n)\left((n \geqq m) \& 0 \leqq s(n)-a(k) / \rho^{k}<1 / \rho^{k}\right) .
$$

\section{THEOREM 1. A rational convergent function has a unique limit.}

We consider first a positive convergent function $s(n)$. The function $s(n)$ is bounded for, by convergence,

$$
(E q)(A n)(s(n) \leqq M(q)+\{(s(q)+1)-M(q)\})
$$

where $M(n)$ satisfies the recursion equations

$$
M(0)=s(0), \quad M(n+1)=M(n)+(s(n+1)-M(n))
$$

(so that $M(n)$ is the greatest of $s(r), 0 \leqq r \leqq n$ ). Thus $s(n)$ has an upper limit $l$, say, such that, for all $k$,

$$
s(m)-(l)_{k}=0(k) \quad \text { for infinitely many } m ;
$$

$l$ is the only limit, for if, for all $k, l^{*}$ satisfies

$$
s(n)-\left(l^{*}\right)_{k}=0(k) \quad \text { for infinitely many } n
$$

then, since by convergence, for all $k$,

$$
s(m)-s(n)=0(k)
$$

for all sufficiently great $m, n$,

we have 


$$
(l)_{k}-\left(l^{*}\right)_{k}=0(k-2)
$$

for all $k$,

whence (anticipating the definition of equality for endless decimals given in 1.4 below) $l=l^{*}$.

It follows from (i) and (ii) that the unique limit $l$ of a convergent function $s(n)$ satisfies the condition: for all $k$ and for all sufficiently great $n$

$$
s(n)-(l)_{k}=0(k-1) .
$$

If $s(n)<0$ for all sufficiently great values of $n$, then $-s(n)>0$, and since $-s(n)$ converges when $s(n)$ converges, therefore $-s(n)$ has a unique limit $l$, say, whence $-l$ is the unique limit of $s(n)$.

If $s(n)$ takes both positive and negative values for arbitrarily great values of $n$, then, if $s(n)$ converges, $s(n)$ has the unique limit zero. We have, for all $k, s(m)-s(n)=0(k)$ for all sufficiently great $m, n$ and $s(p)>0, s(q)<0$ for infinitely many $p, q$ so that, for all $k, s(p)=0(k), s(q)=0(k)$ for infinitely many $p, q$. Thus 0 is a limit of $s(n)$, and, by convergence, it is the only limit.

1.31. We denote the limit of the convergent function $s(n)$ by $\lim s(n)$ or $\lim _{n} s(n)$.

1.4. The arithmetic of endless decimals. If $a=(a)_{n}, b=(b)_{n}$ are endless decimals, then $(a)_{n} \pm(b)_{n}$ and $(a)_{n}(b)_{n}$ are convergent; we define

$$
\begin{aligned}
& a \pm b=\lim \left\{(a)_{n} \pm(b)_{n}\right\}, a b=\lim \left\{(a)_{n}(b)_{n}\right\}, \\
&(A k)\left\{(a)_{k}=0\right\} \leftrightarrow . a=0, \\
&\{a-b=0\} \leftrightarrow . a=b, \\
&(E k)\left\{(a)_{k} \gtrless 0\right\} \leftrightarrow . a \gtrless 0, \\
&\{a-b \gtrless 0\} \leftrightarrow . a \gtrless b .
\end{aligned}
$$

We observe that for any two assigned endless decimals $a, b$ one, and only one, of the relations

$$
a=b, \quad a>b, \quad a<b
$$

holds, for . $(A k)\left\{(x)_{k}=0\right\} . \vee .(E k)\left\{(x)_{k}>0\right\} . \vee .(E k)\left\{(x)_{k}<0\right\}$. is demonstrable for any assigned decimal $x$, in particular for $x=a-b$.

We even have

$$
\begin{aligned}
& .(A k)\left\{(x)_{k}=0\right\} \cdot \bigvee \cdot(E p)\left\{(A k)\left(k \geqq p \rightarrow(x)_{k}>0\right)\right. \\
& \left.\bigvee(A k)\left(k \geqq p \rightarrow(x)_{k}<0\right)\right\} .
\end{aligned}
$$

1.5. If $f(n, \xi)$ is a rational function which converges for any rational number $\xi$ (or any rational $\xi$ in some interval), then we denote $\lim f(n, \xi)$ by $F(\xi)$. The metamathematical sign $F(\xi)$ is called a decimal function of a rational argument.

1.51. As a particular case of 1.5 , if $f(n, m)$ is convergent for all integers $m$, we denote $\lim _{n} f(n, m)$ by $F(m)$. 
1.6. A decimal $l$ is said to be a limit point of a function $F(\xi)$ if we can define a rational function $s(n)$ such that

$$
(A m)(A n)\{(m \neq n) \rightarrow(s(m) \neq s(n))\}
$$

and

$$
(A k)(E p)(A n)\{. n \geqq p . \rightarrow(F(s(n))-l=0(k))\} .
$$

1.7. A decimal function of an integer argument, $F(m)$, is said to be convergent if

$$
(A k)(E n)(A p)\{F(n+p)-F(n)=0(k)\} .
$$

Theorem 2. If $F(m)$ converges then $F(m)$ has a unique limit.

We prove first that $(F(m))_{m}$ converges. By the convergence of $F(m)$,

$$
(A k)(E n)(A p)(A q)\{(p \geqq n) \&(q \geqq n) \rightarrow(F(p)-F(q)=0(k))\}
$$

and so, for $p \geqq q=\max (k, n)$,

$$
\begin{aligned}
\left|(F(p))_{p}-(F(q))_{q}\right| & \leqq\left|(F(p))_{p}-F(p)\right|+|F(p)-F(q)|+\left|F(q)-(F(q))_{q}\right| \\
& <\rho^{-p}+\rho^{-k}+\rho^{-q} \leqq 3 \rho^{-k} .
\end{aligned}
$$

By Theorem 1, $l=\lim (F(m))_{m}$ exists. We show that $l$ is the unique limit of $F(m)$; for we can define $m_{k}$ so that

$$
\begin{aligned}
|l-F(m)| & =\left|l-(F(m))_{m}+(F(m))_{m}-F(m)\right| \\
& <\rho^{-k}+\rho^{-m} \leqq 2 \rho^{-k}
\end{aligned}
$$

if $m \geqq \max \left(k, m_{k}\right)$. The limit of a convergent decimal function of an integer argument, $F(m)$, is denoted by $\lim F(m)$.

1.8. If $F(\xi)$ is a decimal function of a rational argument such that for any assigned decimal $x$ (or any assigned $x$ such that $a \leqq x \leqq b$ for some $a, b$ ) $F\left(s_{n}\right.$ ) converges to a unique limit for all rational sequences $s_{n}$ which converge to $x$, we denote $\lim F\left((x)_{n}\right)$ by $F(x)$.

$F(x)$ is called a decimal function of a decimal argument. We take for granted the extension of the notation of $\$ \S 1.5,1.8$ to functions of several argument places, and also the extension of 1.6 to a function $F$ of a decimal argument (cf. Theorem 4).

$a \pm b$ and $a \cdot b$, given by 1.4 (i) are examples of two-variable functions of decimal arguments.

1.9. If for some integer $\nu,\left|(b)_{\nu}\right|>0$, we define $1 / b$ to be the limit (existing by Theorem 2 ) of the convergent function $1 /(b)_{m}$.

1.91. The upper limit of a bounded rational function. If $m \leqq f(n) \leqq M$ for all $n$, then $0 \leqq|m|+f(n) \leqq|m|+M$, so that, by $1.3,|m|+f(n)$ has an upper limit $l$, say. It follows that $l-|m|$ is a limit of $f(n)$ which is characterised as the upper limit of $f(n)$, and denoted by $\lim \sup f(n)$. 
We observe that $\lim \sup f(n)$ is the greatest limit of a bounded positive $f(n)$ for if $l=\lim \sup f(n)$ and if $l^{\prime}>l$, then $l^{\prime}>(l)_{k}+1 / \rho^{k}$ from some $k$ onwards, and therefore for at most a finite number of values of $n$, is $f(n)$ contained in any small enough interval containing $l^{\prime}$; thus $l^{\prime}$ is not a limit of $f(n)$. It follows that, for any bounded $f(n), \lim \sup f(n)$ is the greatest limit.

\section{Metamathematical attributes.}

2.1. $F(x)$ is continuous for $a \leqq x \leqq b$ if we can define a decimal function $C(x, k)$ such that

$(A k)\{(a \leqq x \leqq X \leqq b) \&(X-x=0(C(x, k))) \rightarrow . F(X)-F(x)=0(k)$.$\} .$

2.2. $F(x)$ is uniformly continuous for $a \leqq x \leqq b$ if we can define a rational integral function $c(k)$ such that

$(A k)\{(a \leqq x \leqq X \leqq b) \&(X-x=0(c(k))) \rightarrow . F(X)-F(x)=0(k)\}$.

2.3. $F(x)$ is differentiable for $a \leqq x \leqq b$ if we can define an endless decimal function $F^{\prime}(x)$ and an integer function $D(x, k)$ such that

$(A k)\{(a \leqq x<X \leqq b) \&(X-x=0(D(x, k)))$

$$
\left.\rightarrow\left((F(X)-F(x)) /(X-x)-F^{\prime}(x)=0(k)\right)\right\} .
$$

3. The classical proofs of the fundamental theorems of analysis are based upon the tertium non datur

$$
\text { . }(A x) P(x) . \vee .(E x) \sim P(x) .
$$

in which the operators are applied to a non-denumerable class, and are therefore not valid in a denumerable system, but it does not follow that the theorems currently derived by these proofs are not demonstrable in the system. On the contrary, as we shall show, all the familiar foundation theorems of classical analysis are demonstrable in a denumerable system.

THEOREM 3. If $F(x)$ is bounded then $F(x)$ is exactly bounded.

We consider first a rational function $f(m, n)$ convergent in $m$ for any $n$ and bounded for all $n$ and all sufficiently great $m$.

We may without loss of generality suppose that $f(m, n)$ is positive.

For all $k$ let $M_{k}$ be the least integer which exceeds $\rho^{k} f(m, n)$ for all $n$ and all sufficiently great $m$, so that $M_{k}-1$ is equalled or exceeded by $\rho^{k} f(m, n)$ for some $n$ and infinitely many values of $m$. If $F(n)=\lim _{m} f(m, n)$ and $M$ is the endless decimal $M_{k} / \rho^{k}$, then $M$ is the exact upper bound of $F(n)$, for $F(n) \leqq M$ for all $n$, and for any $k$ there is an $n$ such that

$$
F(n) \geqq M_{k} / \rho^{k}-1 / \rho^{k}=(M)_{k}-1 / \rho^{k} .
$$

Similarly the lower bound may be defined in terms of the least integer $m_{k}$ which, for all $k$, exceeds $\rho^{k} f(m, n)$ for some $n$ and infinitely many values of $m$. 
If now $F(x)$ is a decimal function of a decimal argument, and if $F(x)$ is bounded for $a \leqq x \leqq b$, then denoting the rationals in $(a, b)$ by $a(n)$ we see that $F(a(n))$ has exact bounds $m, M$ (say). Since $F(x)=\lim F\left((x)_{n}\right)$ and $m \leqq F\left((x)_{n}\right) \leqq M$, it follows that $m, M$ are the exact bounds of $F(x)$.

TheOREM 4. If $F(x)$ is bounded then $F(x)$ has at least one limit.

For any bounded decimal function $G(n)$, the rational function $(G(n))_{n}$ is bounded and so has an upper limit, which is necessarily a limit of $G(n)$. Hence for any rational function $s(n), F(s(n))$ has a limit, and so $F(x)$ has a limit.

THEOREM 5. If $F(x)$ is continuous for $a \leqq x \leqq b$ then $F(x)$ is uniformly continuous for $a \leqq x \leqq b$.

Let

$$
a_{r}^{p}=a+(b-a) r / 2^{p} \quad\left(=\lim _{n}\left\{(a)_{n}+\left((b)_{n}-(a)_{n}\right) r / 2^{p}\right\}\right)
$$

and let $P(k, n, r)$ stand for $\left(a_{r}^{n} \leqq x \leqq_{r+1}^{n}\right) \rightarrow F(x)-F\left(a_{r}^{n}\right)=0(k)$. The formula

$$
(A k)(E n)(A r)\left(0 \leqq r \leqq 2^{n}-1 \rightarrow P(k, n, r)\right)
$$

obviously implies the uniform continuity of $F(x)$. From the contrary

$$
(E k)(A n)(E r)\left(0 \leqq r \leqq 2^{n}-1 \& \sim P(k, n, r)\right)
$$

we derive in turn

$$
\begin{aligned}
k^{*} & =\mu_{z}(A n)(E r)\left(0 \leqq r \leqq 2^{n}-1 \& \sim P(z, n, r)\right), \\
r(n) & =\mu_{z}\left(0 \leqq x \leqq 2^{n}-1 \& \sim P\left(k^{*}, n, z\right),\right.
\end{aligned}
$$

so that $(A n) \sim P\left(k^{*}, n, r(n)\right)$ holds; the bounded function $a_{r(n)}^{n}$ has an upper limit $l$. By continuity at $l$ we can find an interval $i$ containing $l$ such that

$$
F(x)-F\left(x^{\prime}\right)=0\left(k^{*}\right)
$$

for any two $x, x^{\prime}$ in $i$. But the interval $i$ contains the interval $a_{r(n)}^{n}, a_{r(n)+1}^{n}$ for some $n$ so that

$$
a_{r(n)}^{n} \leqq x \leqq a_{r(n)+1}^{n} \rightarrow F(x)-F\left(a_{r(n)}^{n}\right)=0\left(k^{*}\right)
$$

that is, $(E n) P\left(k^{*}, n, r(n)\right)$ holds, and this contradiction establishes the theorem.

THEOREM 6. If $F(x)$ is continuous for $a \leqq x \leqq b, F(x)$ is bounded for $a \leqq x \leqq b$.

This follows from Theorem 5.

THEOREM 7. A continuous function attains its exact bounds. 
By Theorem 6 a continuous $F(x)$ is bounded, and so, by Theorem 3, exactly bounded; let $m, M$ be the exact bounds for $a \leqq x \leqq b$. Defining $a_{r}^{n}$ as in Theorem 5, we have $(A n)(A r)\left\{F\left(a_{r}^{n}\right) \leqq M\right\}$ and so, either $(E n)(E r)$ $\left\{F\left(a_{r}^{n}\right)=M\right\}$, which proves $M$ is attained, or $(A n)(A r)\left\{F\left(a_{r}^{n}\right)<M\right\}$; since $M$ is an exact bound there is an $a_{r}^{n}, \alpha_{k}$ say, such that

$$
(A k)\left\{F\left(\alpha_{k}\right)>M-1 / k\right\} \text {. }
$$

By Theorem 4 there is a function $k(n)$, with $k(n+1)>k(n)$, and a decimal $l$, $a \leqq l \leqq b$, such that $\lim \alpha_{k(n)}=l$. Since $F(x)$ is continuous it follows that $\lim F\left(\alpha_{k(n)}\right)=F(l)$ and therefore $F(l) \geqq M$. Combining this with $F(l) \leqq M$, we conclude that $F(l)=M$, and so on.

TheOREM 8. A continuous function attains any value between any two of its values.

This is proved as in [3, pp. 32-33].

ThEOREM 9. Rolle's TheOREM. If $F(x)$ is continuous for $a \leqq x \leqq b$ and differentiable for $a<x<b$, then there is a $c$ such that $a<c<b$ and $F^{\prime}(c)=0$.

We prove first that $F(x)$ is either constant or not constant, for $a \leqq x \leqq b$. For if $a(n), n \geqq 0$, are the rational numbers between $a$ and $b$ (inclusive) then

$$
\text { . }(A n)\{F(a(n))=F(a(0))\} . \vee .(E n)\{F(a(n)) \neq F(a(0))\} .
$$

so that $F(a(n))$ is either constant or not constant. If $F(a(n))$ is not constant then a fortiori $F(x)$ is not constant.

If $F(a(n))$ is constant then $F(x)$ is constant, since $F(x)=\lim F\left((x)_{n}\right)$ $=F(a(0))$. If $F(x)$ is constant, $F^{\prime}(x)=0$; if $F(x)$ is not constant, $F^{\prime}(x)$ vanishes at the point where $F(x)$ attains its exact bound.

4. The foregoing theorems suffice to show that the theory of Riemann integration may be developed in a denumerable system along classical lines. We shall not follow this development, but shall briefly indicate the construction of an integral of the Cauchy type.

4.1. Subdivision. If $\theta(k)$ is a positive integer function and $\sigma(k, r, n)$ is a rational function, convergent in $n$, such that

$$
(A k)(A n)\left\{. \sigma(k, 0, n)=(a)_{n} . \& \cdot \sigma(k, \theta(k), n)=(b)_{n} .\right\}
$$

and

$$
\begin{aligned}
(A k)(A r)(E p)(E q)(A n)\{. p>0 . \&(. n \geqq q . \rightarrow . \sigma(k, r+1, n) & -\sigma(k, r, n) \geqq 1 / p .)\}
\end{aligned}
$$

and if $s_{\tau}^{k}=\lim _{n} \sigma(k, r, n)$, then $s_{r}^{k}, 0 \leqq r \leqq(k)$, is called a sequence of subdivisions of the interval $(a, b)$.

4.2. A decimal function $F(x)$ is said to be integrable- $U$ over $(a, b)$ if we can define a sequence of subdivisions $s_{r}^{k}, 0 \leqq r \leqq \theta(k)$, such that 


$$
\begin{aligned}
(A k)(A r)\left\{.(0 \leqq r \leqq \theta(k)-1) \&\left(s_{r}^{k}<x<x^{\prime}<s_{r+1}^{k}\right) .\right. \\
\left.\quad \rightarrow . F\left(x^{\prime}\right)-F(x)=0(k) .\right\} .
\end{aligned}
$$

It is readily proved that if $t_{r}^{\boldsymbol{k}}=\left(s_{r}^{\boldsymbol{k}}+s_{\tau+1}^{\boldsymbol{k}}\right) / 2$, then

$$
\sum_{r=0}^{\theta(k)-1} F\left(t_{r}^{k}\right)\left(s_{r+1}^{k}-s_{r}^{k}\right)
$$

is a convergent function of $k$; the limit of this function is called the integral of $F(x)$ and is denoted by $\int_{a}^{b} F(x) d x$.

A function which is integrable- $U$ may be discontinuous at the points $s_{r}^{\boldsymbol{k}}$, $0 \leqq r \leqq \theta(k)$, but is continuous at any other point; in the language of the theory of measure, a function integrable- $U$ shares with a Riemann-integrable function the property of being continuous almost everywhere.

Theorem 10. Darboux's Theorem. If $F(x)$ is integrable- $U$ over $(a, b)$ and if $x_{r}^{k}, 0 \leqq r \leqq N(k)$, is a sequence of subdivisions of $(a, b)$ such that $\lim _{k}\left\{\max \left(x_{r+1}^{\boldsymbol{k}}-x_{r}^{\boldsymbol{k}}\right), 0 \leqq r \leqq N(k)-1\right\}=0$, then, for any $c_{r}^{\boldsymbol{k}}$ in $\left(x_{r}^{\boldsymbol{k}}, x_{r+1}^{\boldsymbol{k}}\right)$,

$$
\lim _{k} \sum_{r=0}^{N(k)-1} F\left(c_{r}^{k}\right)\left(x_{r+1}^{k}-x_{r}^{k}\right)=\int_{a}^{b} F(x) d x .
$$

We omit the proof.

Conclusion. A denumerable system shows the possibility of attaining the fundamental results of classical analysis by means of a finitist superstructure on a classical number theory foundation. A denumerable system, nevertheless, is not coextensive with a classical system. Quite apart from the fact that the general theorems we have described are not formulated within the system itself, and cannot be formulated in a system which contains no variable signs for real numbers, classical analysis is far richer in its variety of admissible function definitions; it suffices to mention the function $F(x)$ $=\lim _{m}\left[\lim _{n}\{\cos (m ! \pi x)\}^{2 n}\right]$ which plays an important part in the classical theory of integration but is not definable in our denumerable system since $F(x)$ is not the limit of $F\left((x)_{n}\right)$ when $x$ is irrational.

\section{BiBLIOGRAPHY}

1. D. Hilbert and P. Bernays, Grundlagen der Mathematik, Berlin, Springer, vol. 1, 1934, vol. 2, 1939.

2. R. L. Goodstein, Function theory in an axiom-free equation calculus, Proc. London Math. Soc. vol. 2 (1945) pp. 401-434.

3. - Mathematical analysis, Oxford, Clarendon, 1948.

4. H. Weyl, Das Kontinuum, Leipzig, 1918. (1938).

5. F. B. Fitch, The consistency of the ramified principia, Journal of Symbolic Logic vol. 3

University COLLEGE, LEICESTER, ENGLAND. 